\title{
THE LUNGS AT BIRTH
}

\author{
BY \\ L. B. STRANG \\ From the Department of Paediatrics, University College Hospital Medical School, London
}

(RECEIVED FOR PUBLICATION JUNE 20, 1965)

In order to obtain insight into disturbances of pulmonary function in newborn infants we have to consider the physiological changes that occur in the lungs at birth. By combining the information obtained from a variety of animal experiments with our clinical observations, we can construct a fairly coherent picture of some of these events and see the ways in which their normal course is disturbed by disease. The first breath of air also marks the beginning of individual life, an abrupt change in the mode of external respiration, and it stands in its own right as a subject of general interest.

The ultimate exchange of oxygen and carbon dioxide between cells and their environment occurs by molecular diffusion, but multicellular forms require a specialized organ of external respiration such as gills, placenta, or lungs, to and from which the gases are transported by a circulation. During foetal life the infant's gases exchange in the placenta between two liquids, the maternal and foetal bloods, but from the moment of birth this function is transferred to the lungs where the exchange is between liquid and gas. In this sense the evolutionary development from water to air is re-enacted each time an infant is born.

The foetal lungs are functionally inert. The alveoli are filled with liquid, and they are perfused by only $10 \%$ of the cardiac output. For the lungs to acquire their function as a gas exchanger a number of changes must take place. A gas-liquid interface has to be formed and the alveoli have to acquire a volume of gas which remains in the lungs even at the end of an expiration, and can be exchanged with the atmosphere by the movements of ventilation. It is also fundamental for normal gas exchange that the gas volume should be evenly distributed throughout the lungs. As the gas that enters the foetal lungs occupies a space formerly filled with liquid, we also have to consider how this liquid is removed.
On the other side of the interface a large increase in blood flow is required. Although the rates at which gases equilibrate in the lung depend on the area, thickness, and composition of the tissues lying between the gas and blood, the amounts exchanged depend on the flow of blood through the lungs. In the adult, pulmonary blood flow equals right ventricular output, but in the foetus most of the cardiac output bypasses the lungs. At the start of breathing an increase in pulmonary flow is achieved by a change in the distribution of cardiac output.

These various changes are all a consequence of the first breath of air, which depends on the activity of the respiratory muscles and the nervous system. The present account, however, is confined to events in the lungs. The formation of an alveolar gas volume, the removal of liquid from the foetal lungs, and the increase in pulmonary blood flow are functionally linked, but they can be separated for descriptive purposes.

\section{Formation of Alveolar Volume}

FIG. 1A shows the relation between inflating pressure and lung volume when the lungs of a mature foetal lamb are inflated with air through the trachea. The same relationships apply when the inflating pressure is a negative pleural pressure and have been verified in a variety of other species. During inflation, an opening pressure of $18 \mathrm{~cm}$. $\mathrm{H}_{2} \mathrm{O}$ is required before any air enters the lungs and then they inflate readily to their full volume with only a small further increase in pressure. The deflation curve is widely separated from the inflation curve, and at each pressure much more air remains in the lung during deflation than during inflation. Even at zero pressure about $25 \%$ of the lung volume remains. The second inflation of this lung is different because no opening pressure is now required and the lung inflates to $80 \%$ of its volume at a pressure that was 


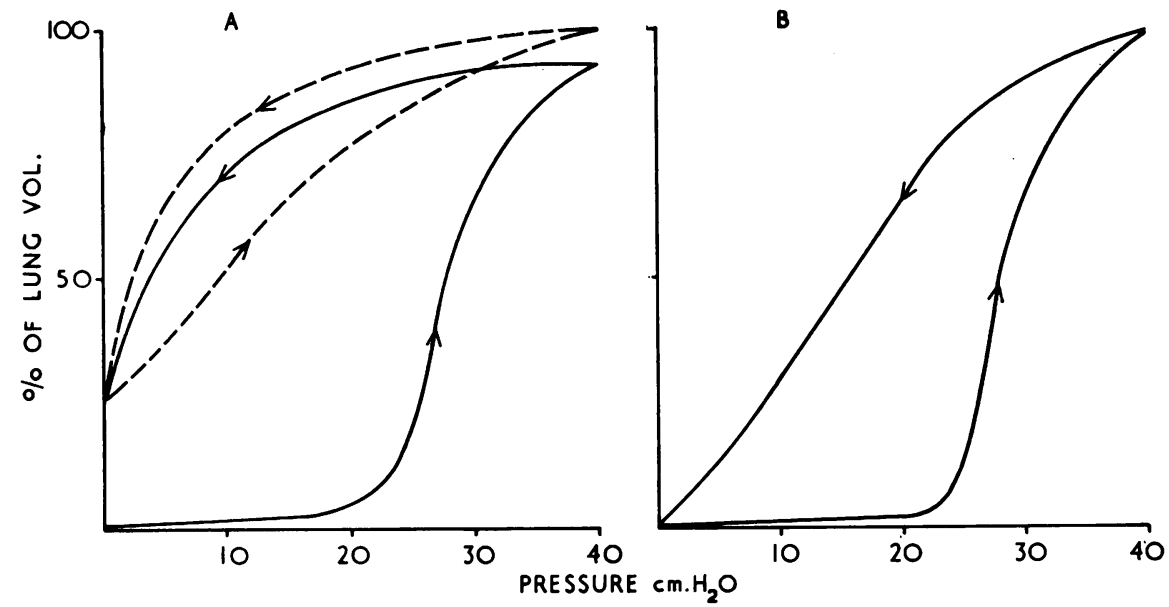

FIG. 1A.-Relationship between inflating pressure and the volume of gas in the lung during the first and second inflation of the lungs of a lamb of 140 days' gestation (term $=147$ days). The first inflation and deflation are shown with a solid line, the second with an interrupted line. Direction (whether inflation or deflation) is indicated by arrows.

Fig. 1B.-Inflating pressure and gas volume of the lungs of a lamb of 122 days' gestation. In this case there is no difference between the first and second inflations.

insufficient to open it for the first time. Fig. 1B shows the relationship for an immature lamb. The inflation curve is similar to Fig. 1A, but the deflation limb is closer to the inflation curve, and at zero pressure the lung is empty of air. For this reason the lung has to be reopened with the second breath and with each succeeding breath. Figs. 2A and 2B represent these curves pictorially as steps in the inflation and deflation of an imaginary alveolus.

Thus in the mature lung a stable alveolar volume (Functional Residual Capacity-FRC) is formed after the first inflation, and normal ventilation can proceed with relatively small pleural pressure changes. Each succeeding tidal breath exchanges about $30 \%$ of this FRC for fresh air (Strang and McGrath, 1962), while oxygen and carbon dioxide exchange continuously between the gas and blood. In the immature lung, as almost no FRC is formed, ventilation requires abnormally large pressures to open the lungs with each new breath, and it seems likely that gas exchange can take place only during inspiration, while the alveoli are held open, instead of during the whole ventilatory cycle. A dynamic picture of ventilation in these two types of lung can be obtained by watching the surface of a lamb's lung during positive pressure ventilation. In the mature lung the first inflation converts the liver-like lung of the foetal lamb to the pale pink inflated appearance

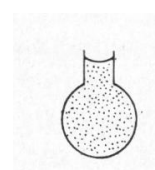

O MATURE

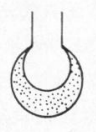

o

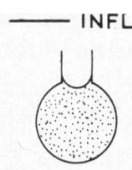

15

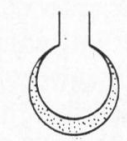

15

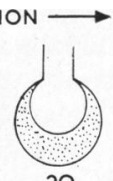

20

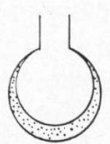

20
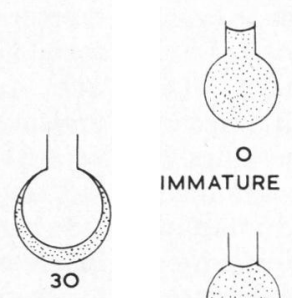

○

IMMATURE

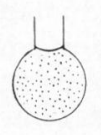

O

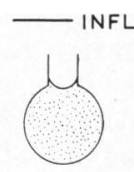

15
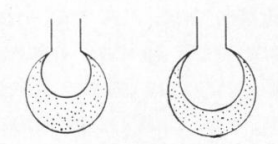

15

$$
\longleftarrow \text { DEFLATI }
$$

Fig. 2A.-Pictorial representation of stages during the initial inflation and deflation of an imaginary alveolus in a mature lung such as that represented in 1A. The alveolus is pictured as being filled with liquid (shaded) before air enters it, and the air is accommodated partly by displacement of the liquid. Numbers indicate inflating pressure in $\mathrm{cm}$. $\mathrm{H}_{2} \mathrm{O}$.

Fig. 2B.-Inflation and deflation of imaginary alveolus from immature lung such as that represented in $1 B$. 
characteristic of normal lungs, and during deflation this appearance is retained. By contrast the immature lung has the inflated appearance only at the peak of inspiration and returns to the foetal appearance during each expiratory phase.

The formation of a stable FRC in the mature lung can be explained by the properties of the surface film which forms in the alveoli. The alveoli of the normal lung are lined by a lipoprotein film which on compression has a very low surface tension, and prevents the alveoli from collapsing under the pressure generated by their surfaces (Pattle, 1955). To understand this statement we have to consider the effect of surface tension at a curved interface. The molecules of a liquid exert an attracting force on each other. At any depth in the liquid these forces balance, but at the surface there remains an unbalanced intermolecular attraction which creates the surface tension. The effect of this force is to pull the surface to as small an area as possible. When the surface is curved, surface tension will exert a force in the direction of the radius. In a sphere, for example a bubble in water, surface tension will produce a pressure difference across the wall. The relationship between the pressure generated in a sphere by the tension in its wall is given by an expression of La Place, $\mathbf{P}=\frac{2 \gamma}{\mathrm{r}}$ (where $\mathbf{P}$ is pressure, $\gamma$ is tension, and $\mathrm{r}$ is radius). Consider an alveolus into which air has been forced by the first inspiration. If the air space which is formed is spherical with a radius of $5 \times 10^{-3} \mathrm{~cm}$. and the surface tension is 55 dynes $/ \mathrm{cm}$. (the surface tension of most body fluids), the surface will exert a pressure of $22 \cdot 5 \mathrm{~cm}$. $\mathrm{H}_{2} \mathrm{O}$. In other words a negative pleural pressure of this amount would be required to hold the space open, and during expiration, when the inspiratory muscles are relaxed, the space would collapse. In the mature lung a surface film of lipoprotein is formed as soon as air enters the lung which, when compressed, yields a very low surface tension, probably of the order of $1-5$ dynes $/ \mathrm{cm}$., whereas the same film when stretched gives a surface tension value of about 50 dynes $/ \mathrm{cm}$. (Clements, Brown, and Johnson, 1958). During inspiration, while the alveoli are enlarging, a considerable pressure is generated by the lung surface, but as soon as expiration starts and the alveolar surface is compressed a little, the surface tension falls and the retractive pressure generated by the surface becomes much less. For example in the alveolus considered above, a surface tension of 1 dyne per $\mathrm{cm}$. would yield a pressure of about $0.5 \mathrm{~cm} . \mathrm{H}_{2} \mathrm{O}$. The presence of lipoprotein (surfactant), which forms a film with these special characteristics, explains the pressure- volume characteristics of the lung illustrated in Fig. 1A, and in particular the small pressure generated during deflation. The surfactant material could not be demonstrated in the lung illustrated in Fig. 1B, which accounts for its different behaviour during deflation, including the failure to form an FRC.

Surfactant also tends to stabilize the distribution of air in the lungs. Because of the relationship between pressure and radius in the La Place equation, smaller alveoli would develop a higher pressure than larger alveoli and tend to empty into them, if the surface tension remained constant. However, as an alveolus decreases in size, its lining film becomes more compressed and its surface tension falls, thus tending to equalize the pressures in alveoli of differing size and consequently to equalize the alveolar sizes themselves.

Thus, the formation of a stable alveolar volume, and its equal distribution in the lungs, depends on the special properties of the surface film which is formed in alveoli when air first enters the lung. The lipoprotein from which the film is formed is probably present in functionally adequate amounts from about 30 weeks' gestation onwards in the human, but it appears relatively later in the gestations of lambs, rabbits, and mice. (For a review of this question see Strang and Reynolds, 1965.)

\section{Removal of Liquid from the Lungs}

The lungs of the foetus contain clear liquid which, in the foetal lamb, pours readily from the cut end of the trachea or bronchus. Its volume is about $50 \mathrm{ml}$. in a foetal lamb of $3.5 \mathrm{~kg}$. (Howatt, Humphreys, Normand, and Strang, 1965), which is similar to the alveolar gas volume (FRC) in infants of similar weight (Strang and McGrath, 1962). Indeed the resting volume of the lungs, which depends on the position where the elastic recoil of the lungs is balanced by the elastic recoil of the chest wall, should be very similar before and after a breath of air if the surface force is small. With a large surface force the gas volume should be smaller than the liquid volume. Setnikar, Agostoni, and Taglietti (1959) and Adams and Fujiwara (1963) have shown that the lung liquid differs in composition from amniotic liquid and contains much less bicarbonate than a blood transudate, from which we may deduce that it is a lung secretion.

Where does the liquid go when breathing starts? Some can be squeezed out through the mouth during the second stage of labour, or drain by gravity when the newborn infant is held head down. The amount that remains in the lungs must vary with the circumstances of delivery and should be greatest after caesarean section. As the survival of mature 
infants after this mode of delivery is as good as after vaginal delivery (Strang, Anderson, and Platt, 1957) the volume drained from the lungs is probably unimportant for survival. The remaining fluid must be taken up when breathing starts, but there have been no direct studies of the rate at which this takes place. The mean weight of mature lambs' lungs falls by about $50 \mathrm{~g}$. during the first six hours of life and then no further, suggesting that the liquid is cleared in this period (author's unpublished data). The liquid is usually assumed to be absorbed directly into the blood, but no change that would favour this transfer, such as a fall in pulmonary capillary pressure, is known to occur. Indeed the pulmonary vasodilatation at the onset of breathing should cause a rise in capillary pressure, if, as seems likely, the change in vasomotor tone is in precapillary arterioles.

Recent experiments have shown that a large increase in lymph flow from the lungs accompanies the onset of ventilation in mature lambs, and it seems likely that some of the lung liquid is cleared from the lungs by this route (Boston, Humphreys, Reynolds, and Strang, 1965). The mechanism envisaged is that air entering the alveoli displaces liquid (as in Fig. 2A) into the interalveolar spaces of the lung where lymphatic capillaries are abundant. A similar mechanism for the removal of lung liquid was suggested by Aherne and Dawkins (1964) from the histological appearance of rabbits' lungs. Boston et al. (1965) found a much smaller increase of lymph flow at the onset of ventilation in immature lambs. In the immature lung it may be that abnormally high surface tensions cause the alveoli to fill up with liquid during expiration (as pictured in Fig. 2B) and impede the transfer of liquid to the interalveolar spaces.

\section{Increase in Pulmonary Blood Flow}

During foetal life most of the inflow from the great veins bypasses the lungs. About half the flow from the inferior vena cava passes directly through the foramen ovale to the left atrium, the left ventricle, and the aorta. The remaining inferior vena caval flow and the superior vena caval flow enters the right atrium, right ventricle, and pulmonary artery. From the pulmonary artery most of the flow goes to the aorta through the ductus arteriosus, and only a small fraction (say $10 \%$ ) passes through the lungs. This distribution depends on the vascular resistance in the lungs being higher than the systemic vascular resistance. Dawes, Mott, Widdicombe, and Wyatt (1953) showed that a large decrease in pulmonary vascular resistance occurred when the lungs were first ventilated and that pulmonary blood flow increased by a factor of 5 or 10 . Closure of the valve of the foramen ovale by a rise in left atrial pressure above inferior vena caval pressure is a consequence of the increased inflow to the left atrium from the lungs. Closure of the ductus arteriosus occurs by active constriction of the muscle in its wall, which contracts in response to a rise in the oxygen tension of the arterial blood leaving the newly ventilated lung. For a review of these changes see Dawes (1958). Thus all the changes in the circulation depend on the decrease in pulmonary vascular resistance at the onset of ventilation, and hence it is important to determine by what means the vascular resistance is lowered.

In experiments that aim to compare the vascular resistance of the lungs under various conditions, serious difficulties of interpretation may arise because the relationship between pulmonary artery pressure and flow is non-linear. Fig. 3 shows two pressure

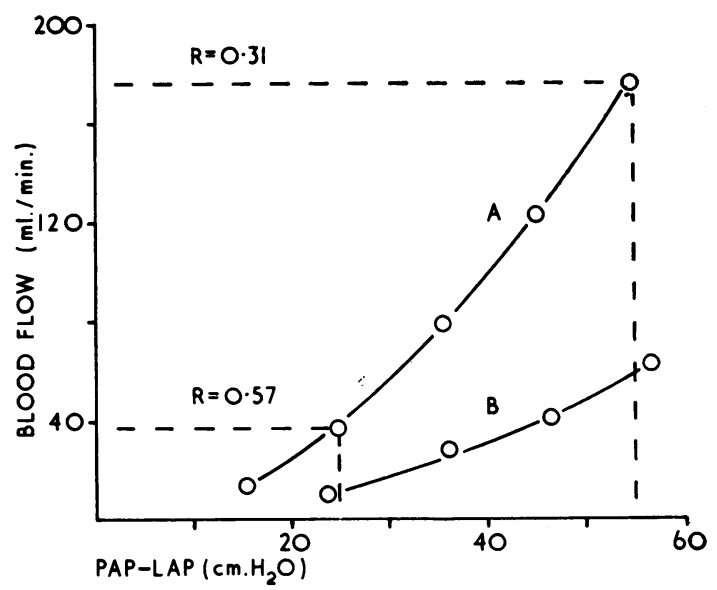

Fig. 3.-Pressure flow curves of the vasculature of the left lung of a lamb. Curve B was obtained during lung collapse and curve $\mathbf{A}$ during ventilation with air. The curves were obtained by perfusing the lung at a series of pulmonary artery pressures (PAP) while left atrial pressure (LAP) remained steady. As shown, calculated 'resistance' (R), obtained by dividing pressure by flow, gives lower values at a high PAP than at a low PAP. In one set of experiments differences such as that between curves $A$ and $B$ were investigated by comparing the flows at a constant PAP. In another set of experiments the comparison was made by drawing pressure flow curves using the device shown in Fig. 5. In Fig. 6 pressure flow curves have been simplified by showing straight lines fitted to the steepest part of the curves. (Reproduced from Cook et al. (1963) by permission of J. Physiol. (Lond.).)

flow curves for the pulmonary vessels of a lamb. If the relationship were linear through zero, resistance, computed as pressure divided by flow, would be a constant, but this is not true of these grossly nonlinear relations. As shown in Fig. 3, a change in perfusion pressure such as will occur during a change in vascular resistance, unless it is artificially controlled, can cause an apparent change in vascular resist- 
ance when there is really no intrinsic change in the vessels themselves. For this reason, in the type of experiments to be considered now, some device has to be adopted that will allow comparisons between states of the vasculature, such as those depicted by the two curves in Fig. 3. There are three possible solutions: (i) to hold flow constant by some device and compare perfusion pressure, (ii) to hold pressure constant and compare flows, (iii) to draw some kind of pressure flow curve for each set of conditions. In the experiments to be described methods (ii) and (iii) have been used.

Experiments in which the left pulmonary artery of the lamb was perfused at a constant pressure showed that the pulmonary vessels could exhibit an extremely active vasomotor tone, and that lowering the oxygen tension or increasing the carbon dioxide tension of the ventilating gas had a strong vasoconstrictor effect (Fig. 4) (Cook, Drinker, Jacobson, Levison,

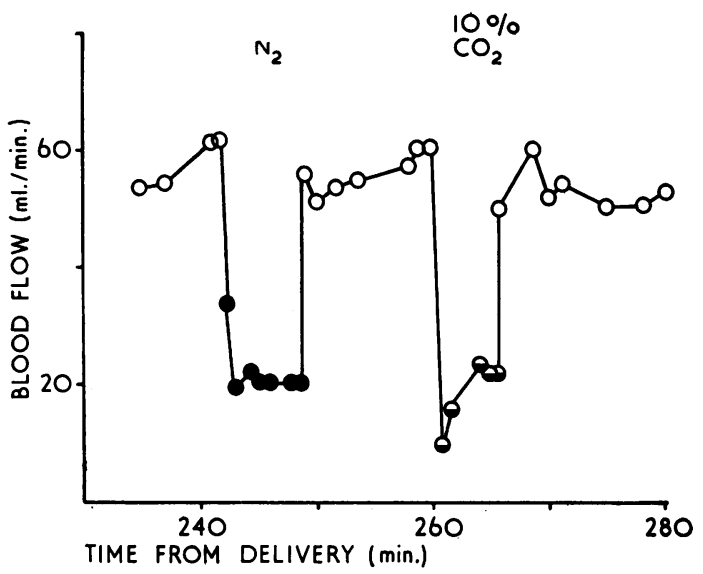

Fig. 4.-Measurement of left pulmonary artery flow in the lamb at a constant pulmonary artery pressure of $40 \mathrm{~cm} . \mathrm{H}_{2} \mathrm{O}$. The open circles are blood flow during ventilation of the lung with air, the closed circles are flow during ventilation with nitrogen, and the half-closed circles are flow during ventilation with $10 \%$ carbon dioxide in air. The sharp decreases in flow at a constant perfusion pressure are due to powerful vasoconstriction. (Reproduced from Cook et al. (1963) by permission of J. Physiol. (Lond.).)

and Strang, 1963). The vasomotor tone of the foetal lung was also detected by the vasodilator effects of acetyl choline and histamine (Dawes and Mott, 1962). The vasoconstrictor effect of asphyxial gas tensions could also be produced in the unexpanded foetal lung by umbilical cord occlusion (Dawes and Mott, 1962) or by perfusing the lung with blood of high $\mathrm{PCO}_{2}$ and low $\mathrm{Po}_{2}$ (Cassin, Dawes, Mott, Ross, and Strang, 1964). There was some evidence that the changes in gas tension were effective mainly by a direct action on the pulmonary vascular muscle, rather than through chemoreceptors (Cook et al., 1963).
The $\mathrm{PO}_{2}$ and $\mathrm{PCO}_{2}$ of the foetal lung are determined by the tensions of these gases in the pulmonary arterial blood. This implies that the areas with the highest $\mathrm{PO}_{2}$ 's will have a tension between 20 and 30 $\mathrm{mm}$. $\mathbf{H g}$, and the areas with the lowest $\mathbf{P C O}_{2}$ 's, a tension between 40 and $50 \mathrm{~mm}$. $\mathrm{Hg}$. Ventilation with air will raise the highest $\mathrm{Po}_{2}$ areas to over $100 \mathrm{~mm}$. $\mathrm{Hg}$ and decrease the $\mathrm{PCO}_{2}$ by an amount dependent on the extent to which blood flow increases in response to ventilation. Plainly the vasodilatation at the onset of breathing could be due to the effects of changes in gas tensions alone. Sensitivity of the vasomotor tone to $\mathrm{PCO}_{2}$ would, in particular, tend to match blood flow to ventilation, because alveolar $\mathrm{PCO}_{2}$ is an almost linear function of the ratio of ventilation to perfusion. For example, if a lung were well ventilated but poorly perfused (high ventilation-perfusion ratio), the $\mathrm{PCO}_{2}$ would fall and tend to increase perfusion by decreasing vasomotor tone.

The extent to which the changes in gas tension do in fact determine the effect of ventilation was investigated by Cassin et al. (1964). In these experiments the vascular 'resistance' of the lamb's lung was determined by drawing pressure-flow curves of the vasculature of the left lung under various conditions by the means described in the captions to Figs. 5 and 6 . The lungs were first ventilated with a mixture of $7 \%$ carbon dioxide in nitrogen which has gas tensions very similar to those of the blood perfusing the foetal lung. In this way the effect of ventilation could be tested independently of the gas tension changes induced by ventilation with air. Subsequently, the ventilating gas was changed to air, or to nitrogen, or to $10 \%$ carbon dioxide in air, so that the effects of increasing $\mathrm{Po}_{2}$ and lowering $\mathrm{PCO}_{2}$ could be measured separately. Fig. 6 gives the maximum slopes of the pressure flow curves obtained, showing the relative effects of ventilation with $10 \%$ carbon dioxide in nitrogen and with the other gas mixtures. In general, $20 \%$ of the vasodilatation was associated with ventilation itself, and the remainder was due in approximately equal amounts to a decrease in $\mathrm{PCO}_{2}$ and an increase in $\mathrm{Po}_{2}$. The means by which ventilation, as distinct from gas tension change, causes vasodilatation is at present unknown, but the effects of $\mathrm{Po}_{2}$ and $\mathrm{PCO}_{2}$ are clearly identified as vasomotor. Naeye (1961) has shown that the muscular coat of pulmonary arterioles in the foetus is thicker than that of the systemic arterioles, and that this muscle atrophies during the first weeks of postnatal life. These arterioles are presumably the site of the observed vasomotor effects.

In summary, the increase in pulmonary blood flow and the redistribution of cardiac output at the start of ventilation are all due to a decrease in pulmonary 


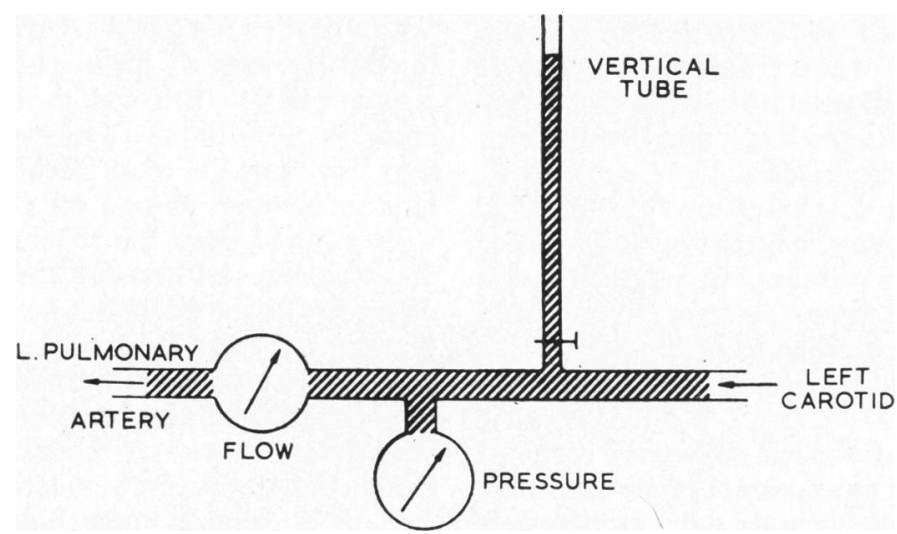

FIG. 5.-Arrangement for obtaining pressure flow curves of pulmonary vasculature in the lamb. The left lung is perfused from the left carotid artery and a vertical tube is placed in the circuit. At intervals this tube is filled with blood and the column of blood is then allowed to flow into the pulmonary artery while the carotid supply is occluded. By recording pressure and flow on the two axes of an oscilloscope or X-Y recorder, a graph of flow over a range of pressures is rapidly obtained. Curves of this kind can be drawn repeatedly. (Reproduced from Cassin et al. (1964) by permission of J. Physiol. (Lond.).)

vascular resistance, which is in large part due to the effects of changing $\mathrm{PO}_{2}$ and $\mathrm{PCO}_{2}$ on the tone of the pulmonary vessels.

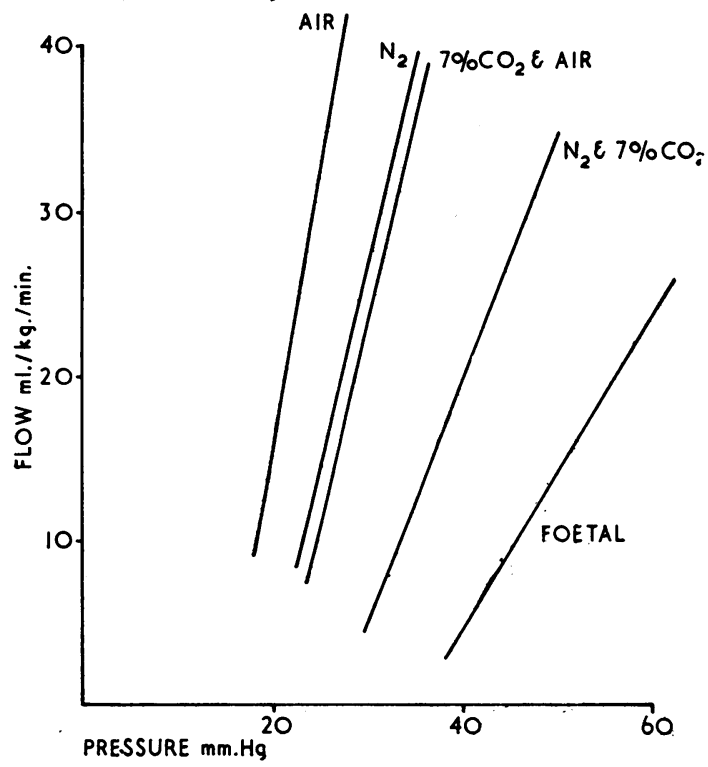

FIG. 6.-Mean pressure flow curves of the left lung of the foetal lamb A straight line has been fitted to the maximum slope of each curve. The lower the vascular resistance, the more vertical the line and the more displaced to the left. The mean curve in the unventilated foetal lung is compared with the mean of the curves obtained after ventilation with a mixture of $7 \% \mathrm{CO}_{2}$ in $\mathrm{N}_{2}$, with $\mathrm{N}_{2}$, with $7 \% \mathrm{CO}_{2}$ in air, and with air as indicated on the diagram. The effect of ventilation unaccompanied by gas tension change is given by the difference between the 'foetal' line and the ' $7 \% \mathrm{CO}_{2}$ in $\mathrm{N}_{2}$ line'. The effect of lowering $\mathrm{PCO}_{2}$ is given by the difference between the $7 \% \mathrm{CO}_{2}$ in $\mathrm{N}_{2}$ ' line and the ' $\mathrm{N}_{2}$ ' line, or by the difference between $7 \% \mathrm{CO}_{2}$ in air' and 'air'. Similarly the effect of raising $\mathrm{PO}_{2}$ is the difference between the ' $\mathrm{N}_{2}$ ' and 'air' lines. (Reproduced from Cassin et al. (1964) by permission of J. Physiol. (Lond.).)

\section{Disturbances of the Physiological Changes in the Lungs at Birth}

We have seen that important changes in the lungs at birth can be traced to two events: the formation of surface film with special properties and an active vasodilatation of pulmonary vessels. When we come to consider cardiopulmonary abnormalities in the newborn we find that the same two factors determine a large part of the abnormalities encountered.

Respiratory Distress Syndrome. Avery and Mead (1959) showed that pulmonary surfactant was absent or inactive in infants dying with hyaline membrane disease. The finding has been confirmed repeatedly (Pattle, Claireaux, Davies, and Cameron, 1962; Reynolds, Orzalesi, Motoyama, Craig, and Cook, 1965b), and hyaline membranes have been shown to develop in immature lambs delivered at 125-130 days' gestation (proportionally equivalent to about 34 weeks in humans), when the alveoli are fully formed, but when pulmonary surfactant cannot be detected by standard techniques (Reynolds, Jacobson, Motoyama, Kikkawa, Craig, Orzalesi, and Cook, 1965a). Indeed the absence of surfactant can account for all the findings in this condition. Extensive atelectasis due to the surfactant deficiency causes the low lung compliance (Gribetz, Frank, and Avery, 1959) which in turn leads to ventilatory insufficiency (Blystad, 1956; Strang and MacLeish, 1961) and to right-to-left shunting (Strang and MacLeish, 1961; Warley and Gairdner, 1962; Prod'hom, Levison, Cherry, Drorbaugh, Hubbell, and Smith, 1962). According to Pattle (1965) the presence of the hyaline membranes, which are 
mainly a blood transudate (Gitlin and Craig, 1956), can also be explained as an effect of a high alveolar surface tension which sets up a pressure gradient between the air space and the underlying capillary during any phase of ventilation in which the alveolus is held open. But variations in capillary permeability may also be an important factor in determining the amount of transudation that occurs. The development of respiratory acidosis is due to the underventilation, and the metabolic acidosis is caused by anaerobic glycolysis due to a deficient oxygen supply, itself the result of the right-to-left shunting. The oxygen deficiency may be aggravated by the excessive oxygen consumption of the overworked muscles of ventilation.

Since the whole chain of events can start with a deficiency of surfactant, the primary deficiency of this substance as a result of immaturity could be the whole cause of the disease. This theory is supported by the prevalence of the disease in prematures, perhaps the only really well-established aetiological factor as yet identified, and by the development of the disease in immature but otherwise undamaged lambs (Reynolds et al., 1965a). On the other hand, the disease usually develops somewhat after the probable time of appearance of surfactant in humans (at about 30 weeks), and it seems likely that other factors can affect the surface, though this has not yet been proved.

Right-to-left Shunting. In general most pulmonary disturbances cause cyanosis by impairment of oxygen diffusion or by inequalities of ventilation with respect to blood flow. The breathing of pure oxygen eliminates these effects by massively increasing the gradient for diffusion and by eliminating the effects of uneven ventilation on alveolar oxygen tensions (for a more rigorous treatment of these statements see Berggren, 1942). Therefore, when cyanosis and arterial desaturation persist while the subject is breathing pure oxygen, it can only be because blood is passing through unventilated areas of the lungs or is bypassing them through extrapulmonary channels.

Newborn infants with ventilatory failure, whether caused by central depression or impaired lung mechanics, often remain deeply cyanosed even in high concentrations of oxygen. In hyaline membrane disease the finding of arterial desaturation in these circumstances is now well established and firmly attributed to right-to-left shunting. Plainly the shunting could be through unventilated atelectatic parts of the lungs, but, in the lamb at least, both lung collapse and underventilation cause an increase of pulmonary vascular resistance, presumably through their effect on alveolar gas tensions (Figs. 7 and 8) (Cook et al., 1963), and this would shunt blood away from the lungs and particularly from any atelectatic areas. Thus it seems more likely to the present author that the large shunts take place through the ductus arteriosus and foramen ovale, i.e. that the circulation returns towards its foetal distribution. Just as a fall in vascular resistance determines the change from a foetal to a neonatal circulation, so will a rise in the resistance reverse the process. The finding of $\mathrm{Chu}$, Clements, Cotton, Klaus, Sweet, Thomas, and Tooley (1965), that excised lungs of infants dying with hyaline membrane disease have a high vascular resistance, considerably strengthens this view of the situation. Indeed the reversibility of the circulatory changes at birth, and

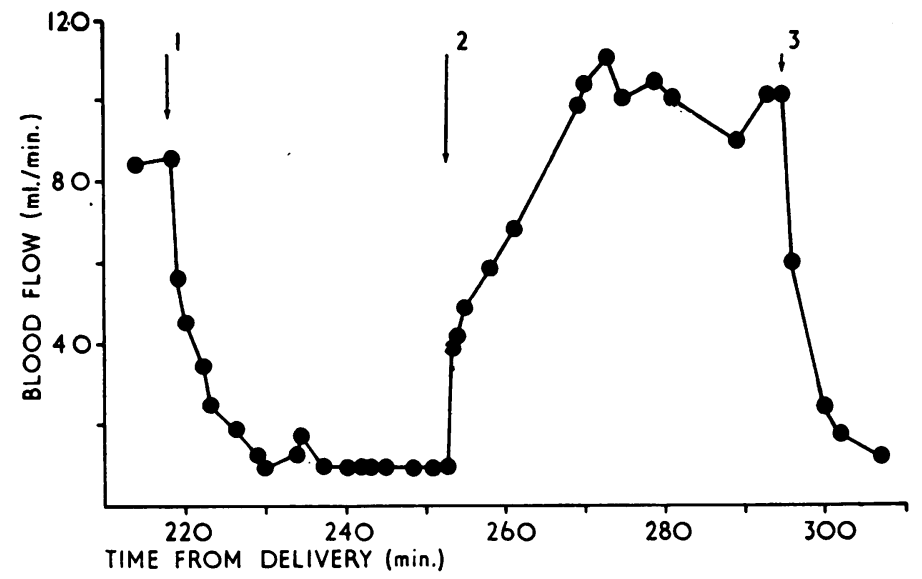

Fig. 7.-Effect of lung collapse in the newborn lamb on blood flow to the left lung at a constant pulmonary artery pressure of $40 \mathrm{~cm}$. $\mathrm{H}_{2} \mathrm{O}$. At the start of the record both lungs are being ventilated with oxygen. At the first arrow ventilation of the left lung is stopped while ventilation of the right lung is continued. The left lung collapses because it contains only absorbable gases. At the second arrow the lung is reventilated, and at the third it is collapsed again by the same procedure. Each episode of lung collapse produces a large reduction of blood flow at constant pulmonary artery pressure, viz. vasoconstriction. (Reproduced from Cook et al. (1963) by permission of J. Physiol. (Lond.).) 


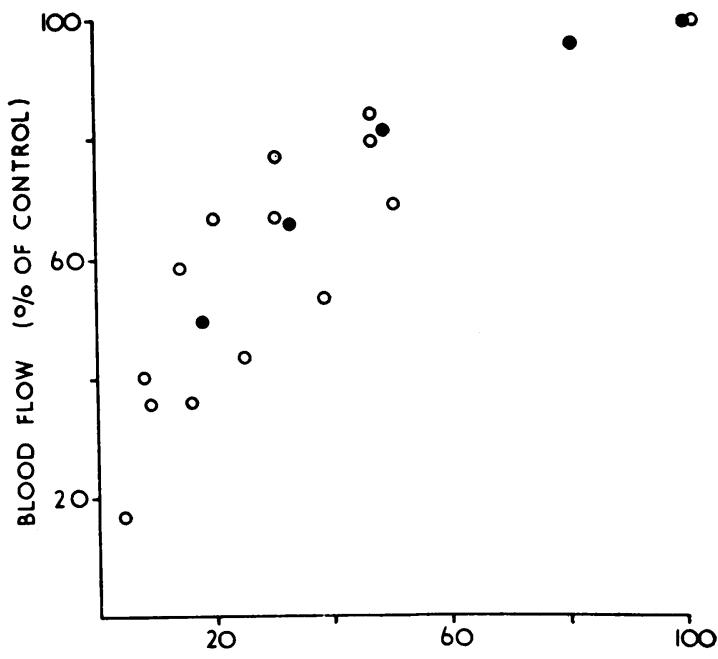

$i_{A}(\%$ OF CONTROL)

FIG. 8.-Effect of changing alveolar ventilation $\left(\dot{V}_{A}\right)$ on blood flow to the left lung at constant pulmonary artery pressure. The open circles represent changes in tidal volume, the closed circles changes in frequency. Reducing pulmonary ventilation causes a more or less proportional reduction in blood flow in these circumstances. (Reproduced from Cook et al. (1963) by permission of J. Physiol. (Lond.).)

their dependence on ventilation of the lungs, means that severe hypoxia is a greater hazard of underventilation in the newborn infant than at other ages - a fact that may well be decisive in determining the high mortality of respiratory conditions in infants.

\section{Summary}

When the normal infant starts to breathe there are at least three important changes in the lungs: they acquire a stable alveolar gas volume, lose the liquid with which they were filled in the foetal state, and increase their blood flow by five or ten times. The formation of the stable alveolar volume depends on the properties of the surface film (surfactant) which forms in the alveoli. The factors concerned in the uptake of liquid are still uncertain. The increase in blood flow is largely due to a decrease in pulmonary vasomotor tone brought about by an increase in $\mathrm{PO}_{2}$ and a decrease in $\mathrm{PCO}_{2}$.

Deficiency or inactivation of surfactant leads to atelectasis and all the abnormalities encountered in the respiratory distress syndrome. Maturity is one important factor in determining the amount of surfactant in the lungs at birth.

Underventilation for any reason causes a rise in pulmonary vascular resistance and a tendency to reverse the changes in the foetal circulation at birth, and hence right-to-left shunting is a common complication of respiratory failure in infants.

\section{REFERENCES}

Adams, F. H., and Fujiwara, T. (1963). Surfactant in fetal lamb tracheal fluid. J. Pediat., 63, 537.
Aherne, W., and Dawkins, M. J. R. (1964). The removal of fluid from the pulmonary airways after birth in the rabbit, and the effect on this of prematurity and prenatal hypoxia. Biol. Neonat., $7,214$.

Avery, M. E., and Mead, J. (1959). Surface properties in relation to atelectasis and hyaline membrane disease. Amer. J. Dis. Child., $97,517$.

Berggren, S. M. (1942). The oxygen deficit of arterial blood caused by non-ventilating parts of the lung. Acta physiol. scand.,4, suppl. 11 .

Blystad, W. (1956). Blood gas determinations on premature infants. III. Investigations on premature infants with recurrent attacks of apnea. Acta paediat. (Uppsala), 45, 211.

Boston, R. W., Humphreys, P. W., Reynolds, E. O. R., and Strang, L. B. (1965). Lymph flow and clearance of liquid from the lungs of the foetal lamb. Lancet, 2, 473.

Cassin, S., Dawes, G. S., Mott, J. C., Ross, B. B., and Strang, L. B. (1964). The vascular resistance of the foetal and newly ventilated lung of the lamb. J. Physiol. (Lond.), 171, 61

Chu, J., Clements, J. A., Cotton, E., Klaus, M. H., Sweet, A. Y. Thomas, M. A., and Tooley, W. H. (1965). The pulmonary hypoperfusion syndrome. Pediatrics, 35, 733.

Clements, J. A., Brown, E. S., and Johnson, R. P. (1958). Pulmonary surface tension and the mucus lining of the lungs: some theoretical considerations. J. appl. Physiol., 12, 262.

Cook, C. D., Drinker, P. A., Jacobson, H. N., Levison, H., and Strang, L. B. (1963). Control of pulmonary blood flow in the foetal and newly born lamb. J. Physiol. (Lond.), 169, 10.

Dawes, G. (1958). Changes in the circulation at birth and the effects of asphyxia. In Recent Advances in Paediatrics, 2 nd ed., ed D. Gairdner, pp. 1-49. Churchill, London.

and Mott, J. C. (1962). The vascular tone of the foetal lung. J. Physiol. (Lond.), 164, 465 .

- - , Widdicombe, J. G., and Wyatt, D. G. (1953). Changes in the lungs of the new-born lamb. ibid., 121, 141.

Gitlin, D., and Craig, J. M. (1956). Nature of the hyaline membrane in asphyxia of the newborn. Pediatrics, 17, 64.

Gribetz, I., Frank, N. R., and Avery, M. E. (1959). Static volumepressure relations of excised lungs of infants with hyaline membrane disease, newborn, and stillborn infants. J. clin. Invest., 38 2168.

Howatt, W. F., Humphreys, P. W., Normand, I. C. S., and Strang, L. B. (1965). Ventilation of liquid by the fetal lamb during asphyxia. J. appl. Physiol., 20, 496.

Naeye, R. L. (1961). Arterial changes during the perinatal period. Arch. Path., 71, 121.

Pattle, R. E. (1955). Properties, function and origin of the alveolar lining layer. Nature (Lond.), 175, 1125.

- (1965). Surface lining of lung alveoli. Physiol. Rev., 45, 48.

- Claireaux, A. E., Davies, P. A., and Cameron, A. H. (1962) Inability to form a lung-lining film: as a cause of the respiratorydistress syndrome in the newborn. Lancet, 2, 469.

Prod'hom, L. S., Levison, H.. Cherry, R. B., Drorbaugh, J. E., Hubbell, J. R., Jr., and Smith, C. A. (1962). Evolution of rightto-left shunt and acid-base balance in newborn infants with respiratory distress. Amer. J. Dis. Child., 104, 465.

Reynolds, E. O. R., Jacobson, H. N., Motoyama, E. K., Kikkawa, Y., Craig, J. M., Orzalesi, M. M., and Cook, C. D. (1965a). The effect of immaturity and prenatal asphyxia on the lungs and pulmonary function of newborn lambs: the experimental production of respiratory distress. Pediatrics, 35, 382.

- Orzalesi, M. M., Motoyama, E. K., Craig, J. M., and Cook, C. D. (1965b). Surface properties of saline extracts of the lungs of newborn infants. Acta paediat. scand. In the press.

Setnikar, I., Agostoni, E., and Taglietti, A. (1959). The fetal lung: a source of amniotic fluid. Proc. Soc. exp. Biol. (N.Y.), 101, 842.

Strang, L. B., Anderson, G. S., and Platt, J. W. (1957). Neonatal death and elective Caesarian section. Lancet, $1,954$.

— , and McGrath, M. W. (1962). Alveolar ventilation in normal newborn infants studied by air wash-in after oxygen breathing. Clin. Sci., 23, 129.

, and MacLeish, M. H. (1961). Ventilatory failure and right-toleft shunt in newborn infants with respiratory distress. Pediatrics, 28, 17.

, and Reynolds, E. O. R. (1965). The alveolar surface properties of the newborn lung. Brit. med. Bull. In the press.

Warley, M. A., and Gairdner, D. (1962). Respiratory distress syndrome of the newborn-principles in treatment. Arch. Dis. Childh., 37, 455. 